\title{
Universal Scaling Behaviour for Iterated Maps in the Complex Plane
}

\author{
N. S. Manton and M. Nauenberg* \\ Institute for Theoretical Physics, University of California, Santa Barbara, CA 93106, USA
}

\begin{abstract}
According to the theory of Schröder and Siegel, certain complex analytic maps possess a family of closed invariant curves in the complex plane. We have made a numerical study of these curves by iterating the map, and have found that the largest curve is a fractal. When the winding number of the map is the golden mean, the fractal curve has universal scaling properties, and the scaling parameter differs from those found for other types of maps. Also, for this winding number, there are universal scaling functions which describe the behaviour as $n \rightarrow \infty$ of the $Q_{n}{ }^{\text {th }}$ iterates of the map, where $Q_{n}$ is the $n^{\text {th }}$ Fibonacci number.
\end{abstract}

\section{Introduction}

During the last few years, considerable progress has been made towards understanding the onset of turbulence or chaos of dynamical systems by studying the properties of one- and two-dimensional maps of real variables. Scaling and renormalization group ideas have elucidated the appearance of universal quantities associated with these maps which are directly relevant to physical systems. In particular, the period doubling transitions have now been found experimentally in various systems with exponents in good agreement with the predictions of the scaling theory [1]. Also progress has been made in applying scaling and renormalization group concepts to one-dimensional maps of the unit circle, which are believed to be generic for transitions which exhibit mode-locking on the route towards chaos [2], and also to the area-preserving twist map relevant to the study of critical Kolmogorov-Arnold-Moser (KAM) trajectories [3]. Other routes to turbulence, such as the scenario described by Ruelle and Takens [4], are understood only qualitatively.

In this paper, we study the scaling properties of a map $z \rightarrow f(z)$, where $z$ is a complex variable and $f(z)$ is complex analytic, and usually a polynomial. Apart from the fact that such a map is a natural extension of the well-studied quadratic map on the unit interval [1], we were motivated by finding numerically that under

* Permanent address : Physics Board, University of California, Santa Cruz, CA 95060, USA 
certain conditions, repeated iteration of the map generates sequences of points lying on closed curves in the complex plane. In fact, the existence of such curves was recognized by Schröder in the last century, although it was only relatively recently that Siegel made Schröder's argument rigorous [5]. We review this theory in Sect. II. Fundamental studies of iterates of rational complex maps were initiated by Fatou [6] and Julia [7], and have continued up to the present time [8-11]. Note that the Schröder theory for complex maps corresponds to Moser's twist theorem, which states sufficient conditions for the existence of a KAM trajectory for area-preserving maps in the plane [5], [12].

Our main results concern the limiting curve beyond which the Schröder theory breaks down. This curve is not smooth, but rather has fractal properties. In Sect. III, we consider the class of maps whose winding number (the average angle of rotation about the origin per iterate) is the golden mean. We present evidence which supports the conjecture that the fractal curves associated with almost all of these maps have universal scaling behaviour. The relationship between this scaling behaviour and the Schröder theory is discussed in Sect. IV. In Sect. V we study the generalization of the period doubling bifurcations which occur for iterated maps in the complex plane.

Finally, a note on our style of presentation: we have direct numerical evidence for almost all the statements in this paper which are not proved. We have presented as Conjectures those of our results which are of greatest generality, and for which it would be most valuable to have an analytic proof.

\section{Schröder Theory and the Fractal}

Let $z \rightarrow f(z)$ be a complex analytic map with a fixed point at $z=0$, and a convergent power series

$$
f(z)=a z+\sum_{j=2}^{\infty} f_{j} z^{j} .
$$

We denote the $n^{\text {th }}$ iterate of $z$ by $f^{n}(z)$, defined inductively by $f^{n}(z)=f\left(f^{n-1}(z)\right)$, $f^{0}(z)=z$. We shall usually take $f$ to be a polynomial, which guarantees the existence of all these iterates. The fixed point is stable if $|a|<1$.

In this section, we assume that $|a|=1$ and $\omega=(2 \pi)^{-1} \arg a$ is irrational. Numerical iteration of the map then leads to a striking result. For any point $z$ sufficiently close to the origin, all iterates of $z$ appear to lie on a smooth curve passing through $z$, which is filled more and more densely as the iteration proceeds. Topologically the curve is a circle surrounding the origin. If the initial point is far from the origin, then the iterates may escape to infinity or there may be a transient behaviour until some iterate lands on one of the invariant curves. Thereafter, all iterates stay on this curve.

The invariant curves very close to the origin are approximately true circles, for then the nonlinearity of $f$ is small, but those further out are less circular, and, remarkably, there is a limiting invariant curve with fractal properties. We shall refer to this curve as the fractal. It seems to be continuous but not differentiable, and the density of points on it, after a fixed number of iterations, is highly non- 
uniform. A key observation is that there is a point on the fractal, denoted by $z_{0}$, which is a stationary point of the map, i.e., $f^{\prime}\left(z_{0}\right)=0$. An example of such a fractal curve, and one of the nearby smooth curves, is shown in Fig. 1. Here $f(z)=a z-z^{2}$, and the winding number is the golden mean $\omega_{0}=\frac{1}{2}(\sqrt{5}-1)$.

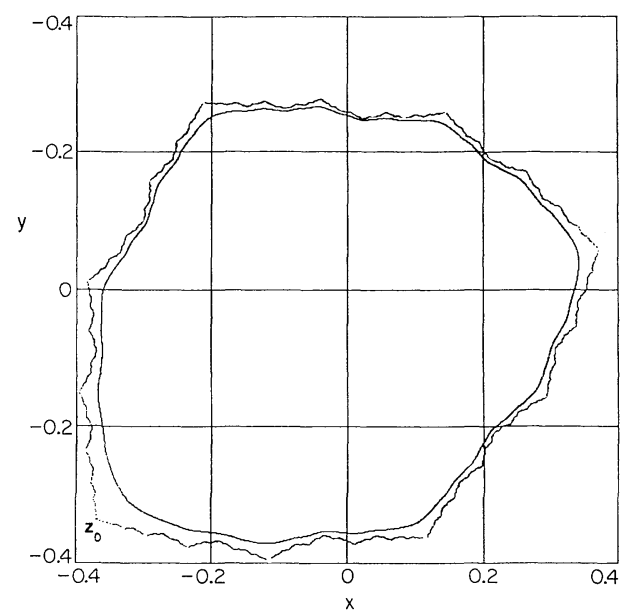

Fig. 1. Fractal and nearby smooth invariant curves for the map $z \rightarrow a z-z^{2}$, where $a=\exp 2 \pi i \omega_{0}$ and $\omega_{0}=\frac{1}{2}(\sqrt{5}-1)$

Some of the above results follow from the classical Schröder theory [5]. Suppose there is an invertible mapping $\phi^{-1}$ from the $z$-plane to a complex $w$-plane such that the effect of $f$ in the $z$-plane is reduced to a simple rotation about the origin in the $w$-plane. Then all iterates of $z$ lie on the image under $\phi$ of a circle in the $w$-plane, hence on a curve. The functional equation for the Schröder function $\phi$ is

$$
f(\phi(w))=\phi(a w) .
$$

In the $w$-plane the rotation per iterate is $\omega$, so the average rotation per iterate, or winding number, of the map $f$ is also exactly $\omega$.

A formal series solution of (2.2) can be obtained. Let

$$
\phi(w)=c w+\sum_{k=2}^{\infty} c_{k} w^{k}, \quad c \neq 0 .
$$

Then (2.1) and (2.2) imply that

$$
\sum_{k=2}^{\infty} c_{k}\left(a^{k}-a\right) w^{k}=\sum_{j=2}^{\infty} f_{j}\left(c w+\sum_{k=2}^{\infty} c_{k} w^{k}\right)^{j} .
$$

The coefficient of $w^{k}$ on the left hand side is $c_{k}\left(a^{k}-a\right)$, whereas the coefficient on the right hand side involves only $\left\{c_{i}: 2 \leqq i \leqq k-1\right\}$. The coefficients $c_{k}$ can therefore be computed sequentially in terms of the coefficients of $f$ and the arbitrary scale parameter $c$. Since $\omega$ is irrational, the denominator $a^{k}-a$ is never zero.

The Schröder series may have zero radius of convergence if $\omega$ is too close to an infinite set of rationals, but Siegel proved the following theorem [5]: 
Theorem. If there exist positive numbers $\lambda, \mu$ (depending on $\omega$ ) such that for all integers $m, n(n>1)$

$$
|\omega-m / n|>\lambda n^{-\mu}
$$

then the Schröder series has finite radius of convergence for all functions $f$ (whose power series (2.1) has finite radius of convergence).

The winding numbers satisfying (2.5) form a set of measure one on the unit interval.

The best way to study rational approximations to the irrational $\omega$ is via the continued fraction representation

$$
\omega=\frac{1}{r_{1}+\frac{1}{r_{2}+\frac{1}{r_{3}+\ldots}}},
$$

where $\left\{r_{i}\right\}$ is a set of positive integers. The rational approximant $p_{n} / q_{n}$ is obtained by truncating the continued fraction at $r_{n}$, and can be computed inductively by

$$
\begin{gathered}
p_{n}=r_{n} p_{n-1}+p_{n-2}, \quad q_{n}=r_{n} q_{n-1}+q_{n-2}, \\
p_{0}=0, \quad q_{0}=1, \quad p_{1}=1, \quad q_{1}=r_{1} .
\end{gathered}
$$

The condition (2.5) fails only if $r_{i}$ increases too rapidly with $i$. In particular, if $r_{i}<R \forall i$, then (2.5) is satisfied with $\mu=2, \lambda=R^{-1}$.

In terms of Schröder theory our main observation can be formulated as the following:

Conjecture 1. For some large class of maps of the form (2.1), the associated Schröder series converges on its circle of convergence, and the image of the circle of convergence is a fractal curve passing through a stationary point of the map.

While it is clear (see below) that the stationary point of the map prevents a larger radius of convergence, it is surprising that we have not encountered a smaller one.

Since a complex analytic function is infinitely differentiable inside its circle of convergence, the invariant curves inside the fractal must be smooth. However, the fractal itself cannot be smooth. To see this, consider the derivative of the Schröder equation (2.2)

$$
f^{\prime}(\phi(w)) \phi^{\prime}(w)=a \phi^{\prime}(a w),
$$

and suppose $z_{0}=\phi\left(w_{0}\right)$. Since $f^{\prime}\left(z_{0}\right)=0$, it follows that if $\phi^{\prime}(w)$ exists at all on the circle of convergence, either $\phi^{\prime}(w)=0$ for all $w=a^{n} w_{0}$, or $\phi^{\prime}(w)=\infty$ for all $w=a^{-n} w_{0},(n>0)$. These image and preimage points $w$ are dense, so the fractal is not even piecewise smooth. We shall conjecture below that the fractal is nowhere differentiable. This argument could fail if $f^{\prime}$ had poles on the curve, but such poles are certainly absent for polynomial maps.

Because the map $f$ is conformal, the neighbourhood of any point on the fractal, other than the stationary point, is geometrically similar to its image under 
$f$. This statement has content even if the curve has neither left nor right derivatives, and hence no corners. For example, if the part of the fractal near $z$ is bounded by lines passing through $z$, then the same is true at $f(z)$, with the same angles between the lines.

Although it appears difficult to explicitly compute the Schröder series (2.3) by the method described earlier, even for the simplest map $f(z)=a z-z^{2}$, we have been able to compute the coefficients numerically. For convenience, we fix the scale parameter $c$ so that $\phi(1)=z_{0}$. The radius of convergence is now unity, assuming that Conjecture 1 is true. Since the fractal is the image of the unit circle

$$
\phi\left(e^{i \theta}\right)=c e^{i \theta}+\sum_{k=2}^{\infty} c_{k} e^{i k \theta},
$$

the coefficients can be obtained from its Fourier transform. In practice, we need values of $\phi\left(e^{i \theta}\right)$ for a uniformly spaced set of angles $\theta$. There is no exact way of getting these, but if we take a rational approximant $p / q$ of $\omega$, and replace $a=\exp 2 \pi i \omega$ by $a=\exp 2 \pi i p / q$, then we can regard $z_{0}$ and the $q-1$ iterates of $z_{0}$ as approximately the set $\phi(\exp 2 \pi i n p / q), 0 \leqq n \leqq q-1$. The angles $2 \pi n p / q$ have uniform spacing $2 \pi / q$. Alternatively, one can leave $a$ unchanged, take $q-1$ iterates, and interpolate.

We have good numerical evidence in just one special case (see Sect. IV), but are prepared to make the following general conjectures about the coefficients.

Conjecture 2. (i) $\sum_{k=2}^{\infty}\left|c_{k}\right|$ converges.

(ii) $\lim _{k \rightarrow \infty}\left|k c_{k}\right|$ does not exist; in particular, it is not zero.

The first of these conjectures has the important consequence that the Schröder series (2.9) converges absolutely and uniformly on the unit circle, so the fractal is continuous. Since $\phi(\exp i \theta)$ is bounded, the interior of the fractal has finite area. The second conjecture prevents term by term differentation of the series and suggests that the fractal is nowhere differentiable.

A general property of $\phi$, which follows from the Schröder equation and the single-valuedness of $f$, is that $\phi(\exp i \theta)$ can equal $\phi\left(\exp i \theta^{\prime}\right)$ for some $\theta \neq \theta^{\prime}$ only if $\phi(\exp i \theta)$ is periodic in $\theta$ with period $2 \pi / N, N>1$. But $\phi$ is $1-1$ on circles close to the origin, so by continuity of the winding number, $N \neq 1$ is impossible. Therefore $\phi$ is continuous and $1-1$ on the unit circle, and its image, the fractal, is a Jordan curve. It follows that its interior is a domain $D$, and $\phi^{-1}$ is the Riemann map from $D$ to the unit disc.

A final conjecture, based on numerical evidence for one particular map discussed in Sect. III, is that the fractal has infinite length, and a dimension $d>1$.

\section{Scaling Properties}

In this section we describe results obtained when the winding number is the golden mean $\omega_{0}=\frac{1}{2}(\sqrt{5}-1)=0.618034 \ldots$ The golden mean has the continued fraction 
representation

$$
\omega_{0}=\frac{1}{1+\frac{1}{1+\frac{1}{1+\ldots}}},
$$

and its rational approximants are of the form $Q_{n} / Q_{n+1}$, where $Q_{n}$ is the $n^{\text {th }}$ Fibonacci number. Siegel's theorem guarantees that the Schröder series has finite radius of convergence for this winding number.

We have worked mainly with maps $f(z)=a_{0} z-z^{2}+C z^{3}$, where $a_{0}=\exp 2 \pi i \omega_{0}$, and in particular with the quadratic map $(C=0)$. The fractal curves associated with them exhibit scaling behaviour which is independent of $C$ (except for some special values to be discussed), and we believe that this same behaviour is universal to a much larger class of maps, including almost all polynomials whose constant term vanishes and whose linear term is $a_{0} z$, and for which $f^{\prime}(z)$ has just simple roots.

When $z$ lies inside the fractal curve, the Schröder function is differentiable, so the sequence of Fibonacci iterates of $z, f^{Q_{n}}(z)$ approaches $z$ along a smooth curve at a rate determined by the winding number $\omega_{0}$. We have $Q_{n} \omega_{0}=Q_{n-1}-\left(-\omega_{0}\right)^{n}$. Hence, for large $n$, if $z=\phi(w)$, then

$$
f^{Q_{n}}(z)=\phi\left(e^{2 \pi i Q_{n} \omega_{0}} w\right),
$$

so

$$
f^{Q_{n}}(z)-z \simeq i\left(-\omega_{0}\right)^{n} c_{1}(z)+\omega_{0}^{2 n} c_{2}(z)
$$

where

$$
\begin{gathered}
c_{1}(z)=-2 \pi w \frac{d \phi}{d w}, \\
c_{2}(z)=-2 \pi^{2}\left(w \frac{d \phi}{d w}+w^{2} \frac{d^{2} \phi}{d w^{2}}\right) .
\end{gathered}
$$

Note, in particular, that

$$
\lim _{n \rightarrow \infty} \frac{f^{Q_{n+1}}(z)-z}{f^{Q_{n}}(z)-z}=-\omega_{0} .
$$

When $z$ lies on the fractal, the derivatives of $\phi(w)$ do not exist and we expect a different behaviour. Consider first the stationary point of the map $z_{0}$, which lies on this curve. In contrast with (3.3), for large $Q_{n}$ (we have gone up to $n=28$ ) we find

$$
f^{Q_{n}}\left(z_{0}\right)-z_{0} \simeq c_{+} \alpha^{n} e^{i(-)^{n} \theta_{+}},
$$

where $\alpha=0.741932 \ldots, 2 \theta_{+}=107 \cdot 27^{\circ} \ldots$, and $c_{+}$is a complex constant. A more precise way of expressing this result is

$$
\left.\lim _{\substack{n \text { even } \\ n \text { odd }}}\right\}_{n \rightarrow \infty} \frac{f^{Q_{n+1}}\left(z_{0}\right)-z_{0}}{f^{Q_{n}}\left(z_{0}\right)-z_{0}}=\alpha e^{\mp 2 i \theta_{+}} .
$$


Because the map $f$ is conformal everywhere on the fractal except at $z_{0}$, Eq. (3.7) is also valid if $z_{0}$ is replaced by any preimage of $z_{0}$. However, assuming that $f^{\prime \prime}\left(z_{0}\right) \neq 0$ (which excludes some values of the cubic coefficient $C$ ), at the image of $z_{0}$ we have

$$
\lim _{\substack{n \text { even } \\ n \text { odd }}} \frac{f^{Q_{n+1}}\left(f\left(z_{0}\right)\right)-f\left(z_{0}\right)}{f^{Q_{n}}\left(f\left(z_{0}\right)\right)-f\left(z_{0}\right)}=\alpha^{2} e^{\mp 4 i \theta_{+}},
$$

and this scaling behaviour is propagated to all succeeding iterates of $z_{0}$.

Since both the images and preimages of $z_{0}$ are dense on the fractal, this type of scaling behaviour excludes the possibility that the fractal is even piecewise differentiable. It is also not surprising that the scaling behaviour (3.5) on the smooth curves is intermediate between that of (3.7) and (3.8), i.e. that

$$
\alpha^{2}<\omega_{0}<\alpha, \quad 2 \theta_{+}<\pi<4 \theta_{+} .
$$

The Fibonacci preimages of $z_{0}$ [denoted $\left.f^{-Q_{n}}\left(z_{0}\right)\right]$ are obtained by iterating the inverse map $f^{-1}(z)$. When $f(z)=a_{0} z-z^{2}$,

$$
f^{-1}(z)=\frac{a_{0}}{2}-\left(\frac{a_{0}^{2}}{4}-z\right)^{1 / 2}
$$

with the branch cut directed outside the fractal, and $f^{-1}(0)$ set to zero. Defined this way, $f^{-1}$ has the same invariant curves, including the fractal, as $f$. We find that

$$
f^{-Q_{n}}\left(z_{0}\right)-z_{0} \simeq c_{-} \alpha^{n} e^{-i(-)^{n} \theta_{-}},
$$

where $2 \theta_{-}=119.6^{\circ} \ldots$ and $c_{-}$is a new constant. This scaling behaviour is similar to that of (3.6).

These results are illustrated in Fig. 2a, which shows part of the fractal close to $z_{0}$ for the quadratic map. The points where the fractal and the lines with opening angle $2 \theta_{+}$intersect are Fibonacci iterates of $z_{0}$ (the number of iterates is indicated). The Fibonacci preimages of $z_{0}$ are where the fractal intersects the lines with opening angle $2 \theta_{-}$.

These observations concerning Fibonacci iterates are special cases of a more general approximate scaling property of invariant curves near $z_{0}$, including the fractal. We find that the fractal is self-similar after a uniform expansion about $z_{0}$ by the scale factor $\alpha^{-1}$, and a reflection in the symmetry axis shown in Fig. 2a, which bisects the opening angle between the two lines generated by the Fibonacci iterates of $z_{0}$. Fig. $2 \mathrm{~b}$ shows part of the curve in Fig. $2 \mathrm{a}$, but expanded by $\alpha^{-1}$. In Fig. 2c, this is further reflected in the axis of symmetry and brought into coincidence with Fig. 2a. (The small offset is for clarity.) Furthermore, a segment of a smooth curve through a point $z_{0}+\eta$ on the symmetry axis coincides, after the same rescaling and reflection, with a segment of the curve through the point $z_{0}+\eta / \alpha$, provided $|\eta| \ll 1$.

The approximate self-similarity of the fractal near $z_{0}$ enables us to define a new non-closed curve. Thus $z_{0}+\delta$ lies on this new curve if there is a sequence of points $\left\{z_{0}+\delta_{n}\right\}$ on the fractal such that $\lim _{n \rightarrow \infty} \delta_{n} / \alpha^{2 n}$ exists and equals $\delta$. Heuristically, the resulting curve is like Fig. $2 \mathrm{a}$, bounded between the lines shown, but stretching to 

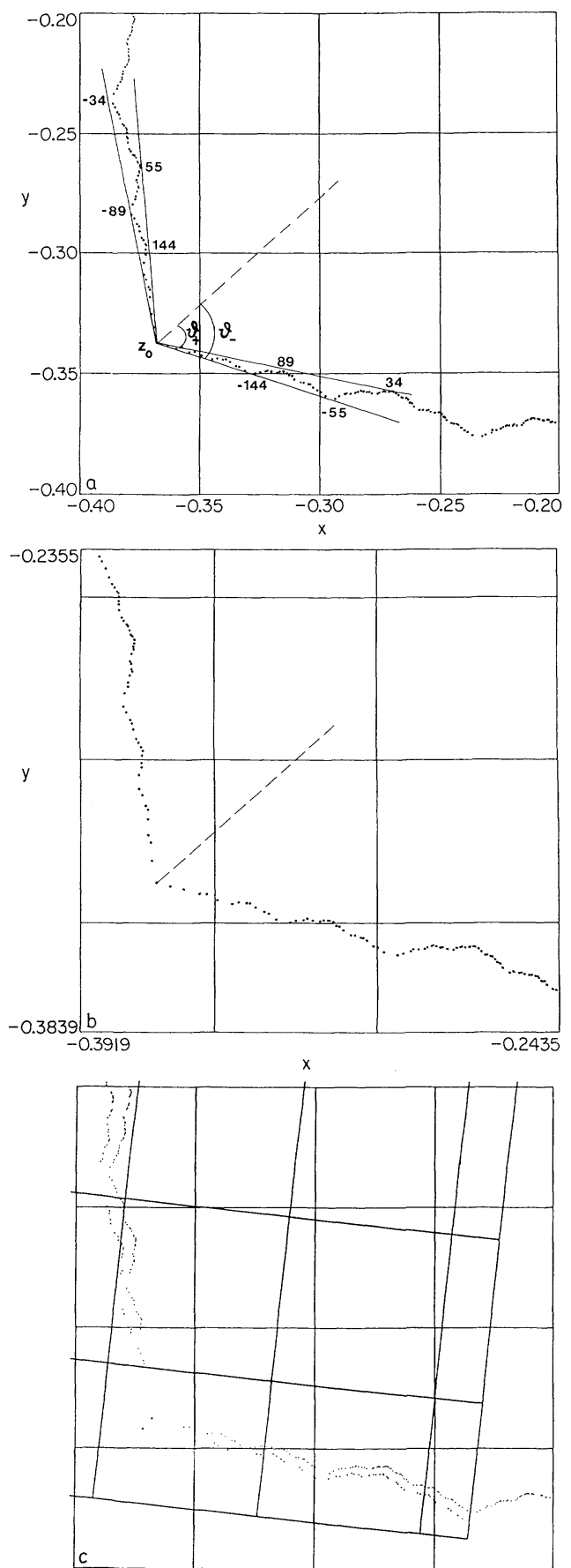

Fig. 2a. Part of the fractal of Fig. 1, close to $z_{0}$, and some Fibonacci iterates of $z_{0}$. Positive numbers denote forward iterates and negative numbers denote iterates of the inverse map. The dashed line is the symmetry axis. b Same as a, but rescaled by $\alpha^{-1}$. c Superposition of b, after a reflection in the symmetry axis, and a. This shows the self-similarity of the fractal near $z_{0}$ 
infinity. It is exactly self-similar. This curve is important because we have evidence that up to a linear transformation, it is universal, occurring for almost all maps with winding number $\omega_{0}$. We shall therefore refer to it as the universal fractal.

We now present a generalized scaling result involving Fibonacci iterates of any point $z$ lying on an invariant curve and close to $z_{0}$. This has been verified numerically to high accuracy in a certain domain. To start it is convenient to introduce a new variable $\xi=\left(z-z_{0}\right) e^{-i \chi}$, where $\chi$ is the angle between the symmetry axis and the real axis. Under a reflection in this axis, $\xi \rightarrow \xi^{*}$. Now define $F^{n}(\xi)=\left[f^{n}\left(z_{0}+e^{i \chi} \xi\right)-z_{0}\right] e^{-i \chi}$. Our result is that for large $Q_{n}$ and small $|\xi|$,

$$
\alpha F^{Q_{n}}(\xi) \simeq\left(F^{Q_{n+1}}\left(\alpha \xi^{*}\right)\right)^{*} .
$$

When $\xi=0$, this relation leads to Eq. (3.6).

The general form of the solution to Eq. (3.12) is

$$
F^{Q_{n}}(\xi) \simeq \alpha^{n}\left[g_{1}\left(\xi / \alpha^{n}\right)+(-)^{n} i g_{2}\left(\xi / \alpha^{n}\right)\right],
$$

where $g_{1}$ and $g_{2}$ are real analytic functions which will be referred to as scaling functions. More precisely, we define the scaling functions as a limit (which we believe exists), namely

$$
\left.\lim _{\substack{n \text { even } \\ n \text { odd }}}\right\}_{n \rightarrow \infty} \frac{F^{Q_{n}}\left(\alpha^{n} \xi\right)}{\alpha^{n}}=g_{1}(\xi) \pm i g_{2}(\xi) .
$$

It is clear that they are defined on the universal fractal and on the half-space to one side of it corresponding to the fractal's interior. We conjecture that the scaling functions themselves are universal.

To recover Eq. (3.7), it is necessary that

$$
g_{1}(0)=c_{+} \cos \theta_{+}, \quad g_{2}(0)=c_{+} \sin \theta_{+} .
$$

On the other hand, suppose that $|\xi|$ is large, but that $z_{0}+\alpha^{n} e^{i \chi} \xi$ is interior to the fractal for all sufficiently large $n$. Now we have made a crossover from the scaling associated with the fractal to the scaling associated with the smooth invariant curves, and Eq. (3.3) is satisfied provided that as $|\xi| \rightarrow \infty$,

$$
g_{1}(\xi) \simeq \xi+\frac{\beta_{1}}{\xi^{p_{1}}}, \quad g_{2}(\xi) \simeq \frac{\beta_{2}}{\xi^{p_{2}}}
$$

with

$$
p_{1}=2 p_{2}+1, \quad p_{2}=\frac{\ln \omega_{0}}{\ln \alpha}-1=0.612 \ldots
$$

Note that for $z$ near $z_{0}$, Eqs. (3.16) and (3.17) also give the $z$ dependence of the functions $c_{1}$ and $c_{2}$ occuring in (3.3) as a power law with coefficients determined by $\omega_{0}$ and $\alpha$.

Because of the defining property of Fibonacci numbers $Q_{n}+Q_{n+1}=Q_{n+2}$, there are two composition rules, namely

$$
\begin{aligned}
& F^{Q_{n+1}}\left(F^{Q_{n}}(\xi)\right)=F^{Q_{n+2}}(\xi), \\
& F^{Q_{n}}\left(F^{Q_{n+1}}(\xi)\right)=F^{Q_{n+2}}(\xi) .
\end{aligned}
$$


We can use these to obtain two functional equations for the scaling functions. Let us first change notation slightly, and define $g(\xi)=g_{1}(\xi)+i g_{2}(\xi)$. Then $g^{*}\left(\xi^{*}\right)=g_{1}(\xi)$ $-i g_{2}(\xi)$, because $g_{1}$ and $g_{2}$ are real analytic. From (3.18) and (3.14) we deduce (for $n$ either even or odd) that

$$
g(\xi)=\frac{1}{\alpha} g^{*}\left(\frac{1}{\alpha} g^{*}\left(\alpha^{2} \xi\right)\right)
$$

and from (3.19) and (3.14), that

$$
g(\xi)=\frac{1}{\alpha^{2}} g\left(\alpha g^{*}\left(\alpha \xi^{*}\right)\right)
$$

We do not know if these equations are sufficient to essentially determine $g$. However, they correspond to Shenker's equations for maps on the unit circle when $\xi$ and $g(\xi)$ are real [3]. Asymptotically, the form of $g$ given by (3.16) satisfies (3.20) and (3.21) provided the exponents satisfy (3.17) and also $\beta_{1}=\frac{1}{2} p_{2} \beta_{2}^{2}$.

Let us now summarize the main results of this section as the following conjecture :

Conjecture 3. When the winding number of the map $f$ is the golden mean, the shape of the invariant fractal near $z_{0}$, the map's stationary point, is characterized by a universal self-similar curve. The Fibonacci iterates of points close to $z_{0}$ have a scaling behaviour which enables one to define a scaling function $g$ on one of the halfspaces bounded by the universal curve, and there are a pair of functional equations which may essentially determine $g$.

Another scaling property concerns the length of the fractal curve. We have found numerically that the length increases as the number of points increases, in such a way that we can associate with the fractal a definite dimension $d>1$. For $d$ to have an unambiguous value, we must take the $Q_{n}(+1)$ points $\left\{f^{i}\left(z_{0}\right):-\left[\frac{1}{2} Q_{n}\right] \leqq i \leqq\left[\frac{1}{2} Q_{n}\right]\right\}$, i.e. an equal number of forward and backward iterates from the stationary point of the map. These points have a well-defined order given by the order of their images on the unit circle under the inverse Schröder map. Joined up in this order they form a polygonal curve, whose length $L_{n}$ defines the length of the fractal for $Q_{n}$ points. We find that

$$
\lim _{n \rightarrow \infty} \frac{L_{n+1}}{L_{n}}=r \sim 1.013 \ldots
$$

A simple definition of the dimension $d$ is suggested by this procedure. Let $\varepsilon_{n}=L_{n} / Q_{n}$ denote the average distance between adjacent points, and let us require that $\lim _{n \rightarrow \infty} Q_{n} \varepsilon_{n}^{d}$ exist. Since $Q_{n} / Q_{n+1} \sim \omega_{0}$, we obtain

$$
d=\frac{\ln \omega_{0}}{\ln \omega_{0}+\ln r} \sim 1.028 \ldots
$$

To conclude this section, we note that the quadratic map has two associated inverse maps corresponding to the two branches of the square root in Eq. (3.10). The region generated from the fractal and its interior by iterating both these 
inverses is a domain whose boundary is the Julia set for the quadratic map. This Julia set is shown in Fig. 3, indicating that it, too, is a fractal. (For reference we have included some points of the original fractal.)

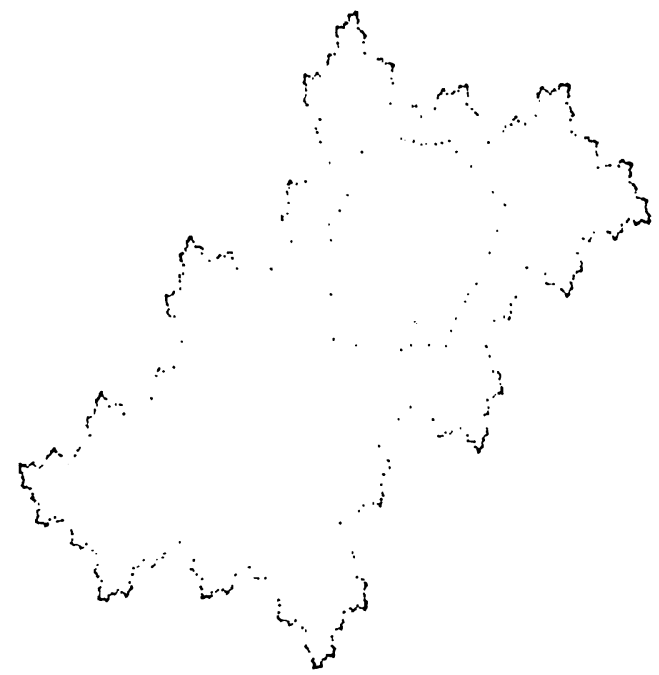

Fig. 3. Julia set for the map whose invariant curves appear in Fig. 1

\section{Scaling and the Schröder Series}

The simplest map $z \rightarrow f(z)$ exhibiting the scaling properties described in the last section is $f(z)=a_{0} z-z^{2}$, where $a_{0}=\exp 2 \pi i \omega_{0}$ and $\omega_{0}$ is the golden mean. Figure 4 is a plot of the absolute values of the coefficients $\left\{c_{n}\right\}$ of the Schröder series (2.3) for

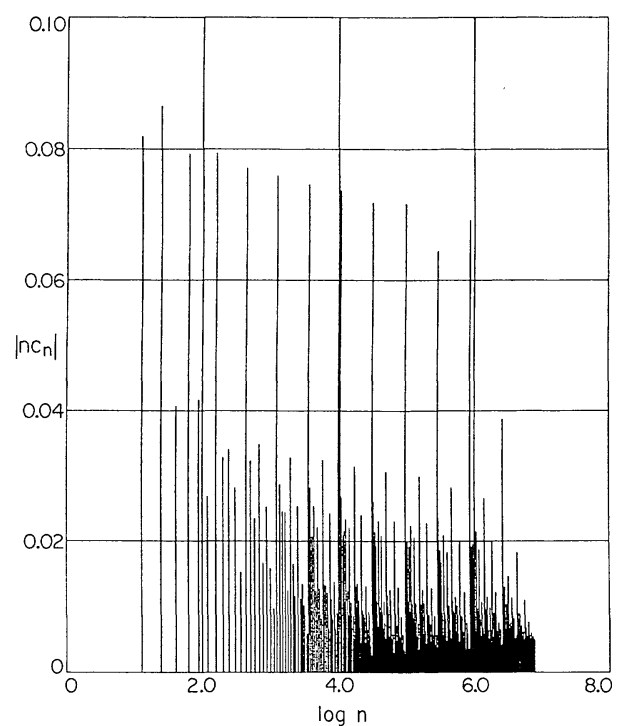

Fig. 4. Absolute values of the Schröder series coefficients for the map whose invariant curves appear in Fig. 1. The data is not reliable for $\log n>6$ 
this map. This data was obtained as described in Sect. II, by Fourier transforming the fractal curve shown in Fig. 1 . The coefficient $c_{n}$ is particularly large when $n-1$ is a Fibonacci number, but this is not surprising, since the denominator $a_{0}^{n}-a_{0}$ is then very small.

The apparent periodicity exhibited in Fig. 4, modified by the increasing density of sample points, suggests the following conjecture:

\section{Conjecture 4.}

$$
\sum_{n=r}^{r+T} c_{n}=\left(\alpha e^{2 i \chi}+O\left(\frac{1}{T}\right)\right) \sum_{n=\left[\omega_{0} r\right]}^{\left[\omega_{0}(r+T)\right]} c_{n}^{*}
$$

for all integers $r$ and $T$ satisfying $\frac{r}{\omega_{0}} \gg r+T \gg r \gg 1$.

Here $\alpha$ is the scaling parameter, and $\chi$ is the angle introduced in Sect. III. $[x]$ denotes the nearest integer to $x$. We require $T \gg 1$, so that $\left[\omega_{0} T\right] / T$ is approximately $\omega_{0}$, but $(r+T) / T$ need not be much greater than 1 .

We have checked the conjecture numerically for a number of values of $r$ and $T$, for the simple quadratic map. However, we believe it is true for all maps whose fractal curve has the universal scaling properties described in the last section.

In what follows we shall argue in the opposite direction. Assuming the conjecture is true, we deduce a fundamental scaling property for the Schröder function $\phi$, from which most of the results of Sect. III follow.

The scaling property of the Schröder function is:

Theorem. For $|\theta| \ll 1, \operatorname{Im} \theta \geqq 0$,

$$
\frac{\phi\left(e^{-i \omega_{0} \theta^{*}}\right)-\phi(1)}{\left[\phi\left(e^{i \theta}\right)-\phi(1)\right]^{*}}=\alpha e^{2 i \chi}+O\left(|\theta|^{\sigma}\right),
$$

where $\sigma>0$.

This theorem will imply scaling behaviour of the fractal ( $\theta$ real), and of the nearby smooth curves $(\operatorname{Im} \theta>0)$, close to the stationary point of the map $(\theta=0)$.

The following is an outline of a proof of (4.2). The argument is formally correct, but the analysis of the error terms is left as an exercise. To get some control over the errors we must assume that

$$
\phi\left(e^{i \theta}\right)-\phi(1)=O\left(|\theta|^{\tau}\right),
$$

where $0<\tau<1$. This is reasonable, since the theorem itself implies that $\tau=\ln \alpha / \ln \omega_{0}$ $=0.62 \ldots$ We can now choose a Fibonacci number $Q_{N}$ such that, for small $|\theta|$,

$$
1 \ll T \ll Q_{N} \ll \frac{1}{|\theta|^{1 / 2(1-\tau)}} .
$$

Then

$$
\phi\left(e^{-i \omega_{0} \theta^{*}}\right)-\phi(1) \simeq \sum_{n=Q_{N}}^{\infty} c_{n}\left(e^{-i n \omega_{0} \theta^{*}}-1\right)
$$


since the terms in the sum with $n<Q_{N}$ approximately cancel. By splitting up the summation into intervals of length $T$, and assuming that the exponential is approximately constant over these intervals, we obtain

$$
\phi\left(e^{-i \omega_{0} \theta^{*}}\right)-\phi(1) \simeq \sum_{p=0}^{\infty}\left(e^{-i\left(Q_{N}+p T\right) \omega_{0} \theta^{*}}-1\right) \sum_{Q_{N}+p T}^{Q_{N}+(p+1) T-1} c_{n} .
$$

Now, use of the conjecture (4.1) and a reorganization of the exponential gives

$$
\phi\left(e^{-i \omega_{0} \theta^{*}}\right)-\phi(1) \simeq \alpha e^{2 i \chi} \sum_{p=0}^{\infty}\left(e^{-i\left(Q_{N-1}+p\left[\omega_{0} T\right]\right) \theta^{*}}-1\right) \sum_{Q_{N-1}+p\left[\omega_{0} T\right]}^{Q_{N-1}+(p+1)\left[\omega_{0} T\right]-1} c_{n}^{*} .
$$

Then, reversing the steps leading to (4.6), but with $Q_{N}$ and $T$ replaced by $Q_{N-1}$ and $\left[\omega_{0} T\right]$, we obtain

$$
\phi\left(e^{-i \omega_{0} \theta^{*}}\right)-\phi(1) \simeq \alpha e^{2 i \chi}\left(\phi\left(e^{i \theta}\right)-\phi(1)\right)^{*},
$$

which proves the theorem.

The self-similarity of the fractal is an immediate consequence of (4.2). Recall that $z_{0}=\phi(1)$ is the stationary point of the map, and suppose $z_{0}+e^{i \chi} \xi=\phi\left(e^{i \theta}\right)$ is on the fractal, with $|\theta| \ll 1$ and $\theta$ real. Then $z_{0}+\alpha e^{i \chi} \xi^{*}=\phi\left(e^{-i \omega_{0} \theta}\right)$, and so this point is also on the fractal.

The scaling properties of the Fibonacci iterates also follow from (4.2). If $z=\phi\left(e^{i \theta}\right)$, then $f^{Q_{n}}(z)=\phi\left(e^{2 \pi i \omega_{0} Q_{n}+i \theta}\right)$. Using the same formula for $\omega_{0} Q_{n}$ as in (3.2), and assuming that $|\theta| \ll 1$, we obtain

$$
\frac{f^{Q_{n+1}}\left(z_{0}+\alpha e^{i \chi} \xi^{*}\right)-z_{0}}{\left[f^{Q_{n}}\left(z_{0}+e^{i \chi} \xi\right)-z_{0}\right]^{*}}=\alpha e^{2 i \chi}+O\left(|\xi|^{\sigma}\right),
$$

for $|\xi| \ll 1$. This result is equivalent to Eq. (3.12).

\section{Bifurcations}

Most of this paper has been concerned with maps of the form (2.1) whose stability parameter $a$ has modulus one. For $|a|<1, z=0$ is a stable, period one, fixed point. Here we show that when $a$ is close to a primitive $n^{\text {th }}$ root of unity, and $|a|$ is just greater than one, there is a bifurcation to a stable cycle of period $n$.

Note that for maps on the real line, only period doubling bifurcations are possible, since $a$ is real. Moreover, such a bifurcation can fail to occur if the equation determining the stable cycle has complex roots. One consequence of working with complex analytic maps is to make these bifurcations more robust.

Suppose first that $a=A$, with $A$ a primitive $n^{\text {th }}$ root of unity $\left(A^{n}=1, A^{m} \neq 1\right.$ for $1<m<n)$.

Lemma.

$$
f^{n}(z)=z+O\left(z^{n+1}\right)
$$

for $z$ in some neighbourhood of zero.

Proof. A modification of the Schröder series construction shows that there is a polynomial $\psi(w)$ of the form

$$
\psi(w)=w+\sum_{k=2}^{n} c_{k} w^{k}
$$


for which

$$
f(\psi(w))=\psi(A w)+O\left(w^{n+1}\right) .
$$

As before, the coefficients $c_{k}$ can be computed sequentially. No higher degree polynomial can be used to reduce the error term in (5.3) because $A^{n+1}-A=0$, so the Schröder series construction would break down. Equation (5.3) implies

$$
f^{n}(\psi(w))=\psi(w)+O\left(w^{n+1}\right) .
$$

Since $\psi$ is invertible near the origin, the lemma follows. It implies that $f^{n}(z)-z$ has an $(n+1)$-fold zero at $z=0$.

The lemma can be checked explicitly for small values of $n$. The coefficients of $z^{2}, z^{3}$, etc. involve $a$ in the combinations $1+a+a^{2}+\ldots+a^{n-1}$, $1+a^{2}+a^{4}+\ldots+a^{2(n-1)}$, etc. These vanish when $a=A$.

Now suppose $a=A(1+\varepsilon)$, where $|\varepsilon|$ is small and $\operatorname{Re} \varepsilon>0$. By continuity (more precisely, by Rouché's theorem) the number of zeros of $f^{n}(z)-z$ near $z=0$ is still $n+1$, and one of them stays at the origin. The remaining $n$ zeros form a closed cycle under iteration by $f$ (since $f^{n}(z)=z \Rightarrow f\left(f^{n}(z)\right)=f(z) \Rightarrow f^{n}(f(z))=f(z)$ etc.).

Let us estimate the locations of the new zeros. Using the lemma, we have

$$
f^{n}(z)=(1+\varepsilon)^{n} z+O(\varepsilon) z+\ldots+\kappa z^{n+1}+\ldots
$$

Assume that $\kappa \neq 0$. To leading order in $\varepsilon, f^{n}(z)-z=0$ when $n \varepsilon z+\kappa z^{n+1}=0$, i.e., when $z=(-n \varepsilon / \kappa)^{1 / n}$ or $z=0$. Provided $|\varepsilon|$ is sufficiently small, $z$ is small and the approximations are valid. In particular, the trajectory of each non-trivial zero $z(\varepsilon)$ has the same tangent vector at $\varepsilon=0$ as that given by the estimate.

To check stability we need to estimate $\left|d f^{n} / d z\right|$ at $z=(-n \varepsilon / \kappa)^{1 / n}$. The leading approximation gives

$$
\frac{d f^{n}}{d z}=1+n \varepsilon+\kappa(n+1) z^{n}=1-n^{2} \varepsilon
$$

at each of the zeros. For suitable $\varepsilon,\left|d f^{n} / d z\right|<1$, so the $n$-cycle is stable.

It is quite interesting to consider values of the stability parameter $a$ for which $|a|=1+\varepsilon(\varepsilon$ now real and positive $)$ and the winding number $\omega$ is irrational, and fixed. For small $\varepsilon$, one may see a stable cycle of period $q$, where $p / q$ is a rational approximant to $\omega$. As $\varepsilon$ decreases, this cycle becomes unstable, but a new stable cycle can appear with longer period $q^{\prime}$, the denominator of a finer rational approximant. This behaviour has been seen numerically. We do not know whether an infinite number of bifurcations can occur as $\varepsilon \rightarrow 0$, nor whether there are gaps between the values of $\varepsilon$ for which there is a stable cycle. However, some aspects of this problem are discussed by Mandelbrot [9].

\section{Conclusions}

We have seen that iterated maps in the complex plane have interesting properties which complement those already known for real one-dimensional maps, and for area-preserving and dissipative maps in the plane. One finds a family of invariant curves enclosing the origin when the origin itself is a marginally stable fixed point 
and the winding number of the map is irrational. This family has a limiting curve which is fractal. When the winding number is the golden mean, the fractal has universal scaling behaviour, but the scaling parameter in this case, $\alpha=0.741932 \ldots$, is different from those associated with the real maps mentioned above.

This difference can be understood if one considers the complex map as a real map in the plane. In polar coordinates $z=r \exp i\left(\theta+2 \pi \omega_{0}\right)$, the quadratic map $f(z)$ $=\left(\exp 2 \pi i \omega_{0}\right) z-z^{2}$ becomes

$$
\begin{gathered}
\theta^{\prime}=\theta+2 \pi \omega_{0}-\arctan \left(\frac{r \sin \theta}{1-r \cos \theta}\right), \\
r^{\prime}=r\left(1+r^{2}-2 r \cos \theta\right)^{1 / 2}
\end{gathered}
$$

For small $r$ this reduces to the "standard" return map on the circle. The Jacobian $J$ of the transformation is

$$
J=\left|f^{\prime}(z)\right|=\left(1-4 r \cos \theta+4 r^{2}\right)^{1 / 2},
$$

so $J>1$ if $r>\cos \theta$, and $J \leqq 1$ otherwise. Hence, from the viewpoint of dynamical systems, this map is neither conservative nor dissipative. Also, the smooth invariant curves of the complex map, while being starlike close to the origin, cease to be so as they approach the fractal. "Starlike" here means that any ray from the origin intersects the curve exactly once. Therefore, for the fractal and the curves nearby, it is not possible to define an effective one-dimensional map from the circle to itself by ignoring the radial coordinate, i.e. by a radial projection onto the unit circle.

We expect that scaling behaviour occurs for complex maps with any winding number whose continued fraction is periodic, and not just for the golden mean. We have some preliminary evidence for this, but further work is needed.

Acknowledgement. We would like to thank G. Culler, S. Ostlund, J. Sethna, and E. Siggia for helpful conversations. We enjoyed also talking with $\mathrm{D}$. Ruelle and would like to thank him for his enlightening comments.

This work is supported by the National Science Foundation, Grant PHY 77-27084.

\section{References}

1. Eckmann, J.-P.: Roads to turbulence in dissipative dynamical systems. Rev. Mod. Phys. 53, 643-654 (1981); Ott, E. : Strange attractors and chaotic motions of dynamical systems. Rev. Mod. Phys. 53, 655-671 (1981); Helleman, R.H.G.: In : Fundamental problems in statistical mechanics, Cohen, E.G.D. (ed.). Amsterdam: North Holland 1980; These are three recent review articles which include references to earlier work

2. Shenker, S.J.: Scaling behaviour in a map of a circle into itself: empirical results. Physica 5D, 405-411 (1982); Ostlund, S., Rand, D., Sethna, J., Siggia, E.: A universal transition from quasiperiodicity to chaos in dissipative systems. Phys. Rev. Lett. 49, 132-135 (1982)

3. Kadanoff, L.P.: Scaling for a critical Kolmogorov-Arnold-Moser trajectory. Phys. Rev. Lett. 47, 1641-1643 (1981); Shenker, S.J., Kadanoff, L.P.: Critical behaviour of a KAM surface: 1. empirical results. J. Stat. Phys. 27, 631-656 (1982); Widom, M., Kadanoff, L.P.: Renormalization group analysis of bifurcations in area-preserving maps. Physica 5D, 287-292 (1982)

4. Ruelle, D., Takens, F.: On the nature of turbulence. Commun. Math. Phys. 20, 167-192 (1971); Newhouse, S., Ruelle, D., Takens, F.: Occurence of strange axiom A attractors near quasi periodic flows on $T^{m}, m \geqq 3$. Commun. Math. Phys. 64, 35-40 (1978) 
5. Schröder, E.: Über iterirte Funktionen. Math. Ann. 3, 296-322 (1871); Siegel, C.L.: Iteration of analytic functions. Ann. Math. 43, 607-612 (1942); For a modern treatment, see Moser, J.K., Siegel, C.L.: Lectures on celestial mechanics. New York: Springer 1971

6. Fatou, P.: Sur les équations fonctionelles. Bull. Soc. Math. France 47, 161-271 (1919), 48, 33-94 and 208-314 (1920)

7. Julia, G.: Mémoire sur l'itération des fonctions rationelles. J. Math. Pures Appl. 4, 47-245 (1918)

8. Brolin, H. : Invariant sets under iteration of rational functions. Ark. Mat. Astron. Pys. 6, 103-144 (1965)

9. Mandelbrot, B.: Fractal aspects of the iteration of $z \rightarrow \lambda z(1-z)$ for complex $\lambda$ and $z$. Ann. N. Y. Acad. Sci. 357, 249-259 (1980)

10. Ruelle, D.: Repellers for real analytic maps. IHES preprint $81 / 52$

11. Douady, A., Hubbard, J.M.: Itération des polynômes quadratiques complexes. C. R. Acad. Sci. (Paris) Sér. 1, 294, 123-126 (1982)

12. Moser, J.: On invariant curves of area-preserving maps of an annulus. Nachr. Akad. Wiss., Göttingen, Math. Phys. Kl. 1-20 (1962)

Communicated by O. E. Lanford

Received June 9, 1982; in revised form January 31, 1983 